\title{
Determining of reservoir fluids properties using PVTP simulation software- a case study of buzurgan oilfield
}

\author{
Tuqa Abdulrazzaq ${ }^{1 *}$, Hussein Togun ${ }^{2}$, Dalia Haider ${ }^{1}$, Mariam Ali $^{1}$, Saja Hamadi ${ }^{1}$ \\ ${ }^{1}$ Department of Petroleum \& Gas Engineering, University of Thi-Qar, 64001 Nassiriya, Iraq \\ ${ }^{2}$ Biomedical Engineering Department, University of Thi-Qar, 64001 Nassiriya, Iraq
}

Keywords: (Fluid properties, PVTP simulator, Reservoir)

\begin{abstract}
The measurement of oil reservoirs and their performance with hydrocarbon reservoirs is used to distinguish the properties of reservoir fluids, which is significant in various reservoir studies. As a result, in the various oil industries, adopting the appropriate methods to obtain accurate property values is very important. The current paper is about a case study of the BUZURGAN Oilfield and how the PVTp software was used to predict phase activity and physical properties. To understand the properties of fluids for the reservoir and phase behavior, the black oil model and the equation of state (EoS) model are used. (Glaso) correlation is used to calculate the bubble point strain, solubility, and formation volume factor. The Beal's correlation was also used to measure viscosity, while the equation of state (EoS) model was used to determine phase behavior and density. Furthermore, the properties of PVT were discovered using the software, and the results were compared to laboratory analysis of PVT, with suitable models being displayed. According to the findings, the used model has the highest saturation pressure, which was chosen for use in reservoir management processes and the preparation of a geological model to reflect the field later. It is clear that the program is appropriate due to the accurate dependence of PVT measurements on laboratory tests in the case that tests are required during the reservoir's productive existence.
\end{abstract}

\section{Introduction}

The measurement of oil reservoirs and their performance with hydrocarbon reservoirs is used to distinguish the properties of reservoir fluids, which is significant in various reservoir studies. As a result, in the various oil industries, adopting the appropriate methods to obtain accurate property values is very important. The current paper is about a case study of the BUZURGAN Oilfield and how the PVTp software was used to predict phase activity and physical properties. To understand the properties of fluids for the reservoir and phase behavior, the black oil model and the equation of state (EoS) model are used. (Glaso) correlation is used to calculate the bubble point strain, solubility, and formation volume factor. The Beal's correlation was also used to measure viscosity, while the equation of state (EoS) model was used to determine phase behavior and density. Furthermore, the properties of PVT were discovered using the software, and the results were compared to laboratory analysis of PVT, with suitable models being displayed. According to the findings, the used model has the highest saturation pressure, which was chosen for use in reservoir management processes and the preparation of a geological model to reflect the field later. It is clear that the program is appropriate due to the accurate dependence of PVT measurements on laboratory tests in the case that tests are required during the reservoir's productive existence.

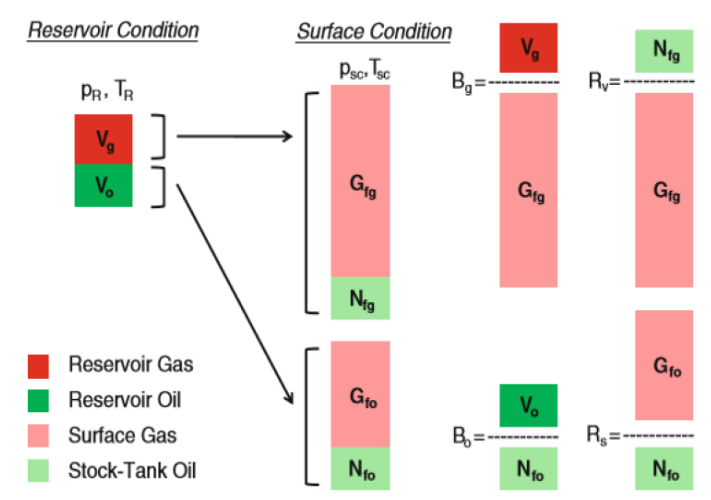

Fig. 1. Graphical representation of standard PVT properties [17]

Since experimental procedures are costly and time consuming, the current paper focuses on a case study of the BUZURGAN Oilfield with predicting phase behavior and physical properties using the PVTp software. Since experimental procedures are costly and time consuming, efforts are devoted to solution equations of state as an alternative approach. 


\section{Buzurgan oilfield}

The Figure 2 illustrated the position of the Buzurgan oilfield, which is located 175 kilometers north of Iraq in the Missan region. The anticline of the Buzurgan oilfield covers $394 \mathrm{~km} 2$ and runs NW-SE parallel to the Zagros Mountain chains, which are $40 \mathrm{~km}$ long and $7 \mathrm{~km}$ wide [1].

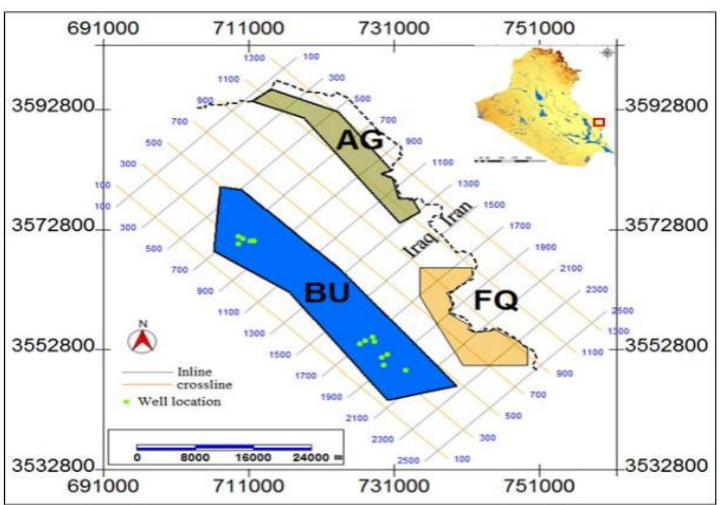

Fig.2. Location Buzurgan oilfield [1]

\section{Mathematical Model}

\subsection{Physical properties of reservoirs fluids}

\subsubsection{Gas solubility}

It is described in SCF/STB [18] as the number of standard cubic feet of gas that dissolve in one stock-tank barrel of crude oil at a given pressure and temperature $R s=\frac{(V g) P i T}{(V o) S c}$

\subsubsection{Gas solubility}

It is defined as the relationship between the volume of oil in a reservoir or separator and the volume of oil under standard conditions.

$(B o)=\frac{(V o)(p ; T) r e s}{(V o) s c}$

The oil formation volume factor is expressed in $\mathrm{bbl} / \mathrm{STB}$ units of reservoir volume over standard volume. The dissolved gas in the oil is lost at the surface due to the high pressure and temperature in the tank. The following setups are generated as a result of the pressure drop from reservoir to surface conditions: (1) Oil weight loss due to dissolved gas loss due to pressure reduction.

(2) As a result of the temperature drop, the oil volume decreases significantly, but the oil slightly expands due to the pressure reduction.

\subsubsection{The viscosity $\mu$}

An applied shearing force can result in a large velocity gradient if the friction between layers of the fluid is low, i.e., low viscosity. Each fluid layer exerts a greater frictional drag on the adjacent layers as the viscosity increases, and the velocity gradient decreases.

$\mu=\frac{\rho u d}{R e}$

\subsection{Black oil modeling}

The solution gas-oil ratio, oil formation volume variables, and viscosity are all common data needed by PVTP for most calculations. Mass conservation and the continuity equation are used to derive the equations for a black oil simulator.

$$
-\nabla \cdot \vec{J}-q=\frac{\partial c}{\partial t}
$$

The fluxes and concentrations of the conservation equations for each of the three phases are used to calculate the flow equations for an oil, water, and gas system. The density of the fluid times its velocity in the given direction can be written as a flux in a given direction. The fluxes are represented by the subscripts $\mathrm{o}, \mathrm{w}$, and $\mathrm{g}$, which represent for oil, water, and gas, respectively.

$\overrightarrow{(J)}_{0}=\frac{\rho_{o s c}}{B_{0}} \cdot \overrightarrow{v_{o}}$

$\overrightarrow{(J)}_{w}=\frac{\rho_{w s c}}{B_{w}} \cdot \overrightarrow{v_{w}}$

$\overrightarrow{(J)}_{g}=\frac{\rho_{g s c}}{B_{g}} \cdot \overrightarrow{v_{g}}+\frac{R_{s o} \rho_{g s c}}{B_{o}} \cdot \overrightarrow{v_{o}}+\frac{R_{s w} \rho_{g s c}}{B_{w}} \cdot \overrightarrow{v_{w}}$

Where $R_{s o}$ and $R_{s w}=$ gas solubilities $B_{o}, B_{w}$, and $B_{g}=$ formation volume factors $\mathrm{Sc}=$ standard conditions (usually $60 \mathrm{~F}$ and $14.7 \mathrm{psia}$ in Oil field units)

$\rho=$ density of fluid

The velocities are Darcy velocities, with the following $\mathrm{x}$ components:

$v_{x o}=-k_{x} \lambda_{o} \frac{\partial}{\partial x}\left[p_{o}-\frac{\rho_{o} g z}{144 g_{c}}\right]$
$v_{x w}=-k_{x} \lambda_{w} \frac{\partial}{\partial x}\left[p_{w}-\frac{\rho_{w} g z}{144 g_{c}}\right]$
$v_{x g}=-k_{x} \lambda_{g} \frac{\partial}{\partial x}\left[p_{g}-\frac{\rho_{g} g z}{144 g_{c}}\right]$

Where $\mathrm{g}$ represents the acceleration of gravity in $\mathrm{ft} / \mathrm{sec}^{2}$ $\mathrm{gc}=32.174 \mathrm{ft} / \mathrm{sec}^{2}$

For the $y$ and $\mathrm{z}$ components, similar expressions can be written.

The relative phase mobility $\lambda_{\ell}$ is equal to the ratio of the phase's relative permeability to flow divided by its viscosity, as follows:

$\lambda_{\ell}=k_{r \ell} / \mu_{\ell}$

By linking phase densities to formation volume factors and gas solubility,

$$
\begin{aligned}
& \rho_{o}=\frac{1}{B_{o}}\left[\rho_{o s c}+R_{S o} \rho_{g s c}\right] \\
& \rho_{w}=\frac{1}{B_{w}}\left[\rho_{w s c}+R_{S w} \rho_{g s c}\right] \\
& \rho_{g}=\frac{\rho_{g s c}}{B_{g}}
\end{aligned}
$$

We also need concentrations in addition to fluxes. These are supplied by:

$C_{o}=\frac{\emptyset \rho_{o s c} S_{o}}{B_{o}}$
$C_{w}=\frac{\emptyset \rho_{w s c} S_{w}}{B_{w}}$ 
$C_{o}=\emptyset \rho_{g s c}\left[\frac{s_{g}}{B_{g}}+R_{s o} \frac{s_{o}}{B_{o}}+R_{s w} \frac{s_{w}}{B_{w}}\right]$

Where $\varnothing$ is defined the porosity

The constraints are satisfied by the saturations.

$s_{o}+s_{w}+s_{g}=1$

A mass conservation equation for each variable in the appropriate phase is obtained by combining equations 4 and 5 into equation 18.

Oil component in the oil phase

$-\left[\frac{\partial}{\partial x}\left(\frac{\rho_{o s c}}{B_{o}} v_{x o}\right)+\frac{\partial}{\partial y}\left(\frac{\rho_{o s c}}{B_{o}} v_{y o}\right)+\frac{\partial}{\partial z}\left(\frac{\rho_{o s c}}{B_{o}} v_{z o}\right)\right]-q_{o}=$ $\frac{\partial}{\partial t}\left(\frac{\varnothing \rho_{o s c} S_{o}}{B o}\right)$

Water component in the water phase

$-\left[\frac{\partial}{\partial x}\left(\frac{\rho_{w s c}}{B_{w}} v_{x w}\right)+\frac{\partial}{\partial y}\left(\frac{\rho_{w s c}}{B_{w}} v_{y w}\right)+\frac{\partial}{\partial z}\left(\frac{\rho_{w s c}}{B_{w}} v_{z w}\right)\right]-$ $q_{w}=\frac{\partial}{\partial t}\left(\frac{\emptyset \rho_{w s c} S_{w}}{B w}\right)$

Gas component in the gas, Oil, and water phases

$-\frac{\partial}{\partial x}\left(\frac{\rho_{g s c}}{B_{g}} v_{x g}+\frac{R_{s o} \rho_{g s c}}{B_{o}} v_{x o}+\frac{R_{s w} \rho_{w s c}}{B_{w}} v_{x w}\right)$

$-\frac{\partial}{\partial y}\left(\frac{\rho_{g s c}}{B_{g}} v_{y g}+\frac{R_{s o} \rho_{g s c}}{B_{o}} v_{y o}+\frac{R_{s w} \rho_{w s c}}{B_{w}} v_{y w}\right)-$

$\frac{\partial}{\partial z}\left(\frac{\rho_{g s c}}{B_{g}} v_{z g}+\frac{R_{s o} \rho_{g s c}}{B_{o}} v_{z o}+\frac{R_{s w} \rho_{w s c}}{B_{w}} v_{z w}\right)-q_{g}=$

$\frac{\partial}{\partial t}\left(\varnothing \rho_{g s c}\left(\frac{s_{g}}{B_{g}}+\frac{R_{S o} S_{O}}{B_{o}}+\frac{R_{S w} S_{w}}{B_{w}}\right)\right)$

The densities at standard conditions are constant, so the preceding equations can be divided. As a result, the equations are reduced to the following form:

Oil component in the oil phase

$-\left[\frac{\partial}{\partial x}\left(\frac{v_{x o}}{B_{0}}\right)+\frac{\partial}{\partial y}\left(\frac{v_{y o}}{B_{0}}\right)+\frac{\partial}{\partial z}\left(\frac{v_{z o}}{B_{0}}\right)-\frac{q}{\rho_{o s c}}\right]=\frac{\partial}{\partial t}\left(\frac{\phi s_{o}}{B_{0}}\right)$

(22)

Water component in the water phase

$$
\begin{aligned}
& -\left[\frac{\partial}{\partial x}\left(\frac{v_{x w}}{B_{w}}\right)+\frac{\partial}{\partial y}\left(\frac{v_{y w}}{B_{w}}\right)+\frac{\partial}{\partial z}\left(\frac{v_{z w}}{B_{w}}\right)-\frac{q}{\rho_{w s c}}\right]= \\
& \frac{\partial}{\partial t}\left(\frac{\phi s_{w}}{B_{w}}\right)
\end{aligned}
$$

Gas component in the gas, oil, and water phases:

$$
\begin{aligned}
& -\frac{\partial}{\partial x}\left(\frac{v_{x g}}{B_{g}}+\frac{R_{s o}}{B_{0}} v_{x o}+\frac{R_{s w}}{B_{w}} v_{x w}\right) \\
& -\frac{\partial}{\partial y}\left(\frac{v_{y g}}{B_{g}}+\frac{R_{s o}}{B_{0}} v_{y o}+\frac{R_{s w}}{B_{w}} v_{y w}\right) \\
& -\frac{\partial}{\partial z}\left(\frac{v_{z g}}{B_{g}}+\frac{R_{s o}}{B_{0}} v_{z o}+\frac{R_{s w}}{B_{w}} v_{z w}\right)-\frac{q_{g}}{\rho_{g s c}} \\
& =\frac{\partial}{\partial z}\left(\phi\left(\frac{S_{g}}{B_{g}}+\frac{R_{s o} S_{0}}{B_{o}}+\frac{R_{s w} S_{w}}{B_{w}}\right)\right)
\end{aligned}
$$

\subsection{Equation of state (EOS)}

The phase Behavior of the reservoir was drawn using the equation of state model, which is used to determine the form of crude oil, saturation pressure, and critical temperature dew point pressure.

The following properties are required by the state equation:

1. Component $(\mathrm{C} 1, \mathrm{C} 2, \mathrm{C} 3, \mathrm{C} 4, \mathrm{C} 5$, pseudo components).

2. Pressure (p).
3. Temperature $(\mathrm{T})$.

4. Solubility (RS).

5. Oil formation volume factor (Bo).

6. Viscosity $(\mu)$.

7. Density $(\rho)$

\section{Technical approach}

After collecting all data related to reservoir fluid properties for the four wells in the Mishrif reservoir, an excel sheet containing the data needed for the software is created for each well. The data is entered into the PVTP program, and the model with the lowest error rate is selected. A match is then made with the input properties, and the program relies on the black oil model, which operates at the cost of the properties of the remaining reservoir fluids.

\section{Results and discussion}

The reservoir fluid properties of an Iraqi oil field called "BUZURGAN" are calculated using the PVTP simulator. As previously described, this simulator employs equation of state and black oil models. The findings of this analysis, which was conducted in order to predict the oil field's phase activity. An important thing to note is the amount of data available on each well, which has a significant impact on the accuracy and reliability of the results obtained. In certain instances, a full collection of analyses may be found on a few of the field's final well reports (BU1, BU6, BU10, BU12). As a result, Table (1) shows the research that is considered representative for each reservoir in this report.

Table 1: List of wells considered

\begin{tabular}{|c|c|c|c|c|c|}
\hline Field & Formation & Well NO. & API gravity & Depth & $\begin{array}{c}\text { Data of } \\
\text { sampling }\end{array}$ \\
\hline BUZURGAN & MISHRIF & BU-1 & 22.7 & $12500 \mathrm{ft}$ & $14 / 1 / 2003$ \\
& & BU-6 & 22.5 & $3910 \mathrm{~m}$ & $2 / 11 / 1976$ \\
& & BU-10 & 22.7 & $3962 \mathrm{~m}$ & $22 / 5 / 1977$ \\
& & BU-12 & 21.7 & $3966 \mathrm{~m}$ & $22 / 4 / 1978$ \\
\hline
\end{tabular}

While the majority of experimentally reported data is of "good quality," this has never eliminated the presence of inaccurate data due to experimental errors. Unrepresentative crude oil sampling and/or human errors caused by laboratory procedures and equipment are also possible causes of errors. To ensure that the data is of good quality and that it is accurate. When the values of certain properties are not similar, such as when extracting the viscosity values, charts are used to approximate them. Using the PVTP tool, several simulations were run to measure the properties of reservoir fluids and compare the measured data to field data.and showed similar behavior for all measured fluid properties, so we'll present and discuss the results of the (BU1) well in this section; the results are shown in (figures 3 to 6). Figure 3 illustrates the variance of Gas Rs instantaneous solubility with pressure $\mathrm{p}$ for BU-1, both for the origin and measured effects. It can be shown that the Rs increases as the pressure rises because the 
gas is forced to solve. This behaviour continues until the pressure exceeds $200 \mathrm{~kg} / \mathrm{cm} 2$, at which point the approximated remains constant. The following scenarios are created as a result of the pressure drop from reservoir to surface conditions: The oil weight decreased as a result of the loss of dissolved gas caused by the pressure drop; the oil volume decreased slightly as a result of the temperature drop; however, the oil slightly expanded as a result of the pressure drop. Temperature and pressure-induced volume contraction and expansion normally balance each other out, resulting in no major changes. The increase in Bo above bubble point pressure corresponds to an increase in oil volume at $\mathrm{P} \& \mathrm{~T}$ and, as a result, an increase in Bo. Oil shrinkage due to gas evolution appears to resolve its expansion below the bubble limit, resulting in pressure reduction, consequently Bo decline steeply42

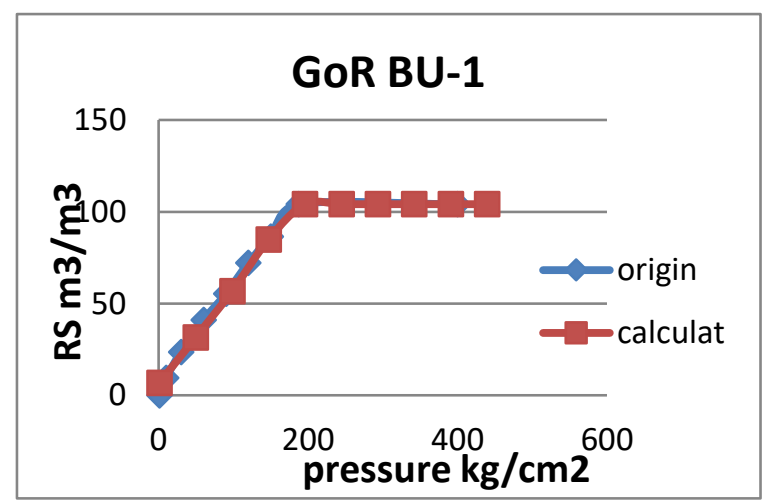

Fig. 3 solubility of gas versus pressure relationship for origin calculate data.

Figure 4 shows the behavior of oil viscosity o as a function of pressure $p$ for BU-1, as well as the measured effects. The viscosity of oil decreases as the pressure rises, as the temperature rises, weakening the particle bonds and lowering the viscosity. Oil viscosity often decreases with decreasing pressure due to decreased friction between fluid layers, and reaches its lowest point at saturation pressure. If pressure is reduced more, more gas escapes from the oil, causing the oil to become denser and more viscous due to the lack of lighter components

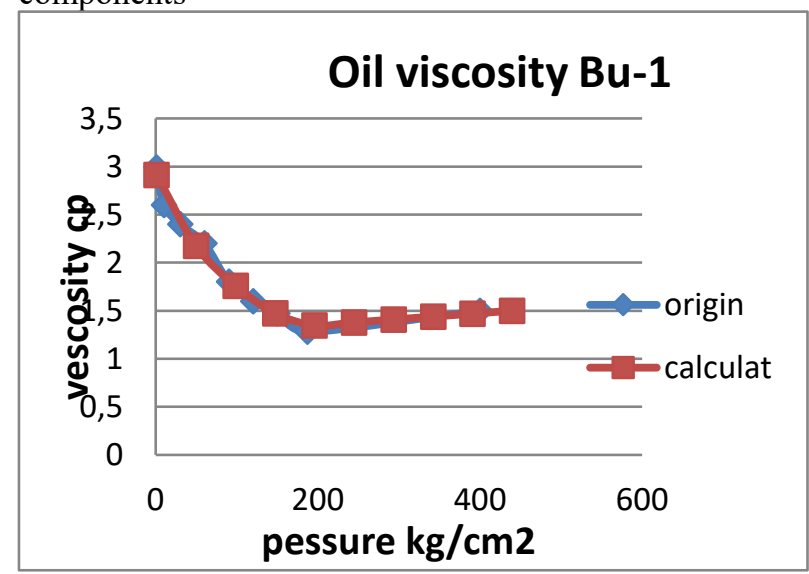

Fig. 4 oil viscosity versus pressure relationship for origin calculate data
Variation in the ratio of oil formation Figure 4 illustrates Bo with pressure for BU-1, as well as the origin and measured effects. Since pressure causes the oil to become more compact and the particles to become closer together, the Bo increases with pressure. This behavior continues until the pressure reaches 200 $\mathrm{kg} / \mathrm{cm} 2$, after which it decreases due to changes in the structure and properties of hydrocarbons at these pressures.

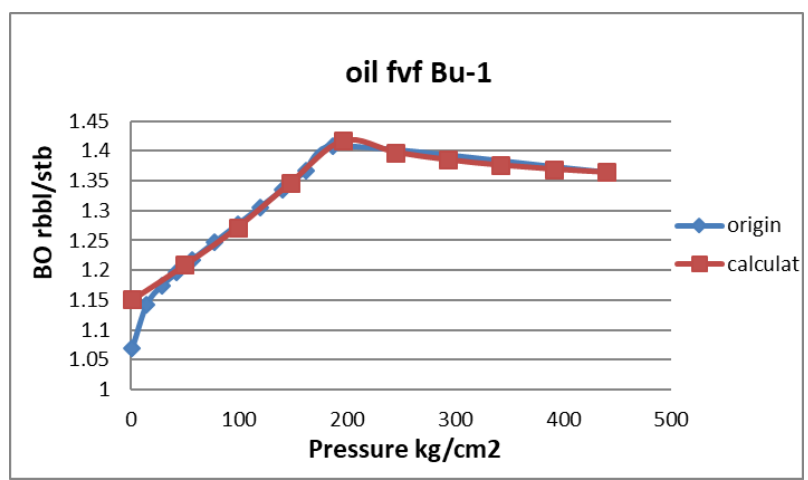

Fig. 5 Oil formation volume factor versus pressure relationship for origin calculate data

Figure 5 describes the relationship between density and pressure, demonstrating the decrease in density values as reservoir pressure rises. This is in direct contrast to what occurs on the surface of the earth. It is natural for the physical properties of oil in the reservoir to vary from those on the surface of the earth since many gases dissolve in the oil and cause a decrease in density as a result of the reservoir being exposed to high pressure and heat.

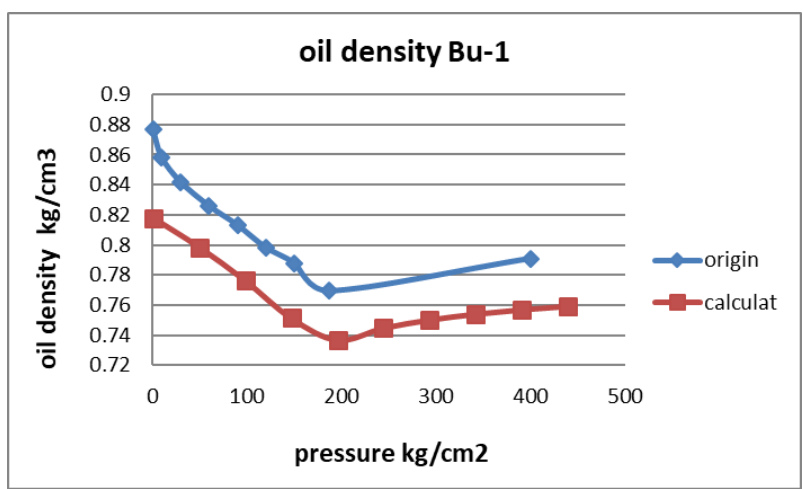

Fig. 6 Oil density versus pressure relationship for origin calculate data

The " PVTP" is a quick and accurate method in calculating the properties of the remaining reservoir fluids by matching to predict the behavior of reservoir fluids by relying on the four ( $\mathrm{P}, \mathrm{RS}, \mathrm{Bo}$, ) characteristics that were entered into the software, as shown in Figure 6 , and the results are shown in Tables (2) and Tables (3). 
Table 3: Data calculated from PVTP software

\begin{tabular}{|c|c|c|c|c|c|c|}
\hline $\begin{array}{c}\text { Oil Density } \\
(\mathrm{g} / \mathrm{cc})\end{array}$ & $\begin{array}{c}\text { Oil } \\
\text { Viscosity } \\
\text { (centipoise) }\end{array}$ & $\begin{array}{c}\text { Oil FVF } \\
(\mathrm{RB} / \mathrm{STB})\end{array}$ & $\begin{array}{c}\text { Gas Oil } \\
\text { Ratio } \\
(\mathrm{m} 3 / \mathrm{m} 3)\end{array}$ & $\begin{array}{c}\text { Bubble } \\
\text { Point } \\
(\mathrm{Kg} / \mathrm{cm} 2 \mathrm{a})\end{array}$ & $\begin{array}{c}\text { Pressure } \\
(\mathrm{Kg} / \mathrm{cm} 2 \mathrm{a})\end{array}$ & $\begin{array}{c}\text { Temperature } \\
(\mathrm{deg} \mathrm{C})\end{array}$ \\
\hline & 2.91244 & 1.13154 & 6.82406 & 187 & 1 & 112 \\
0.817803 & 2.1756 & 1.19397 & 31.6247 & 187 & 49.7144 & 112 \\
0.798509 & 1.75983 & 1.26466 & 56.7588 & 187 & 98.4289 & 112 \\
0.776329 & 1.79435 & 1.34904 & 84.9367 & 187 & 147.143 & 112 \\
0.751371 & 1.47472 & 112 \\
0.736991 & 1.34729 & 1.40477 & 104.12 & 187 & 195.858 & 112 \\
0.744955 & 1.3778 & 1.38975 & 104.12 & 187 & 244.572 & 112 \\
0.750322 & 1.40831 & 1.37981 & 104.12 & 187 & 293.287 & 112 \\
0.754184 & 1.43882 & 1.37274 & 104.12 & 187 & 342.001 & 112 \\
0.757095 & 1.46933 & 1.36747 & 104.12 & 187 & 390.716 & 112 \\
0.759369 & 1.49983 & 1.36337 & 104.12 & 187 & 439.43 & 112 \\
& & & & & & \\
\hline
\end{tabular}

Table 4: Data calculated from PVTP software

\begin{tabular}{|r|r|r|r|}
\hline Gas Density (g/cc) & Gas FVF (ft3/scf) & Z Factor & $\begin{array}{l}\text { Gas Viscosity } \\
\text { (centipoise) }\end{array}$ \\
\hline 0.000843 & 1.33187 & 0.997873 & 0.012885 \\
0.044931 & 0.025002 & 0.901018 & 0.013895 \\
0.097421 & 0.011531 & 0.82275 & 0.016009 \\
0.152532 & 0.007365 & 0.785559 & 0.019223 \\
& & & \\
\hline
\end{tabular}

\section{The conclusions}

The properties of reservoir fluids, which are important in various reservoir studies, oil reservoir calculations, and their performance with hydrocarbon reservoirs, are distinguished in this paper. The main results can be summarized as follows:

-PVT studies are important reservoir engineering experiments that have a wide range of applications, including reserve estimation, surface installations, and storage operations.

-To determine the exact Eos models in the study of PVT, high-quality density and viscosity data are needed.

-The advantages of the computational capabilities of this simulation to estimate the properties of liquids for reservoir hydrocarbon mixtures are evident from this work.

-After drawing all of the wells and comparing the results to the original data tables, the best and most wells were chosen based on the experimental values and the highest bubble point pressure to create the geological model that would reflect the reservoir.

-When performing PVT tests, knowing the bubble point pressure is one of the most critical characteristics to know in order to maintain output at pressures higher than the bubble pressure.

- The model had a high degree of accuracy in terms of adherence to laboratory measurements. This suggests that PVT research procedures and reliance on models can be extended over longer periods of time.

\section{References}

1. Al_Ismaily, Use borehole image log technique to estimate Mishrif Reservoir characterization in Buzurgan oilfield. Unpolished thesis, Ph.D.
Science College, University of Basrah, 206p, (2017).

2. Di Primio R, Dieckmann V, Mills N. PVT and phase behaviour analysis in petroleum exploration. Organic Geochemistry;29(13):207-222, (1998).

3. Mansour E, Farag A, El-Dars F, Desouky S, Batanoni M, Mahmoud M. Predicting PVT properties of Egyptian crude oils by a modified soave-Redlich- Kowng equation of state. Egyptian Journal of Petroleum. 22(1): 137148, (2013).

4. Aplin, A. C., Macleod, G., Larter, S. R., Pedersen, K. S., Sorensen, H., \& Booth, T., Combined use of confocal laser scanning microscopy and PVT simulation for estimating the composition and physical properties of petroleum in fluid inclusions. Marine and Petroleum Geology, 16, 97-110 (1999).

5. Aplin, A. C., Larter, S. C., Bigge, M. A., Macleod, G., Swarbrick, R. E., \& Grunberger, D. (2000). PVTX history of the North Sea's Judy oilfield. Journal of Geochemical Exploration, 69-70, 641-644 (2000).

6. Wei Yan 1, Farhad Varzandeh 2, Erling H. StenbyPVT modeling of reservoir fluids using PC-SAFT EoS and Soave-BWR EoS, Fluid Phase Equilibria 386, 96-124, (2015).

7. Aydelotte, R., Alboudwarej, H., Dindoruk, B., Qi, Y., McCreless, J., Nighswander, J., Kunt, T.A., Lucas-Clements, D., Ormerod, L., Hollingsworth, J., Mao, M., Delivering a PRODML Data-Exchange Standard for PVT and Fluid Characterization Data for the Full Lifecycle of a Reservoir. SPE-173425MS.(2015).

8. Osman, E., Abdel-Wahhab, O.A., AlMarhoun, M., Prediction of Oil Properties Using Neural Networks. Society of Petroleum Engineer. SPE-68233-MS.(2001).

9. Goda, H.M., Shokir, E.M., Eissa, M., Fattah, K.A., Sayyouh, M.H., Prediction of the PVT Data Using Neural Network Computing Theory. SPE-85650-MS. (2003).

10. Numbere, O.G., Azuibuike, I.I., Ikiensikimama, S.S., Bubble Point Pressure Prediction Model for Niger Delta Crude Using Artificial Neural Network Approach. SPE167586-MS.(2013).

11. Ramirez, A.M., Valle, G.A., Romero, F., Jaimes, M., 2017. Prediction of PVT Properties in Crude Oil Using Machine Learning Techniques MLT. SPE-185536-MS (2017).

12. Yang, Q., Jin, B., Banerjee, D., Nasrabadi, H., Direct visualization and molecular simulation of dewpoint pressure of a confined fluid in sub$10 \mathrm{~nm}$ slit pores. Fuel 235, 1216-1223.(2019a).

13. Sinha, U., Dindoruk, B., Soliman, M., Machine learning augmented dead oil viscosity model for all oil types. J. Petrol. Sci. Eng. 195, 107603.(2020).

14. Pouya Hosseinif, Mehdi Assareh, Cyrus GhotbiDeveloping a new model for the 
determination of petroleum fraction PC-SAFT parameters to model reservoir fluids, Fluid Phase Equilibria 412, 145-157, (2016).

15. Ronald ET, Rogers JB. Applied Petroleum Reservoir Engineering. New York: Pearson Education Incorporated.7-10, (2015).

16. Aniedi B. Usungedo and Julius U. Akpabio, Evaluating the Challenges in Pressure Volume -Temperature (PVT) Analysis of Gas Condensate Reservoirs, Journal of Engineering Research and Reports, 16(3): 13-18, (2020).

17. Walsh MP, Lake LW. A Generalized Approach to Primary Hydrocarbon Recovery. Elsevier,Amsterdam.;3- 15, (2003).

18. Vazquez M, Beggs HD. Correlations for fluid physical property prediction. In: SPE Annual Fall Technical Conference and Exhibition. Society of Petroleum Engineers; (1977). 